\title{
Moderately Severe Hallucination
}

National Cancer Institute

\section{Source}

National Cancer Institute. Moderately Severe Hallucination. NCI Thesaurus. Code C156252.

Experiences daily hallucinations, or some areas of functioning are disrupted by hallucinations. 